\title{
Benchmark values for molecular three-center integrals arising in the Dirac equation
}

\author{
A. Bağcl* and P. E. Hoggan \\ Institute Pascal, UMR 6602 CNRS, University Blaise Pascal, \\ 24 avenue des Landais BP 80026, 6317n Aubiere Cedex, France
}

\begin{abstract}
The authors in their previous papers obtained compact, arbitrarily accurate expressions for twocenter one- and two-electron relativistic molecular integrals expressed over Slater-type orbitals. In this present study, the accuracy limits of given expressions is examined for three-center nuclear attraction integrals, which are the first integral set do not have analytically closed form relations. They are expressed through new molecular auxiliary functions obtained via Neumann expansion of Coulomb interaction. The numerical global adaptive method is used to evaluate these integrals for arbitrarily values of orbital parameters, quantum numbers. Several methods, such as Laplace expansion of Coulomb interaction, single-center expansion, Fourier transformation method, have been performed in order to evaluate these integrals considering the values of principal quantum numbers in the set of positive integer numbers. This is the first attempts to study the three-center integrals without any restrictions on quantum numbers and in all ranges of orbital parameters.
\end{abstract}

Keywords:

PACS numbers: ....

\section{INTRODUCTION}

The LCAO-SCF [1] method is generally employed for molecules, in which molecular wave functions taken to be linear combinations of atomic basis functions whose should possess the cusps condition at the nuclei [2] and decay exponentially for large distances [3]. This approach leads to use, namely, Slater-type orbitals $[4,5]$,

$$
\chi_{n l m}(\zeta, \vec{r})=\frac{(2 \zeta)^{n+1 / 2}}{\sqrt{\Gamma(2 n+1)}} r^{n-1} e^{-\zeta r} Y_{l m}(\theta, \phi),
$$

here, $Y_{l m}$ are complex or real spherical harmonics $\left(Y_{l m}^{*}=\right.$ $\left.Y_{l-m} ; Y_{l m} \equiv S_{l m}\right)$ differs from the Condon-Shortley phases by sign factor $(-1)^{m}[6-8], \Gamma(z)$ are gamma functions [9], $\{n, l, m\}$ are the principal, orbital, magnetic quantum numbers with, $n \in \mathbb{R}^{+}, 0 \leq l \leq\lfloor n\rfloor,-l \leq$ $m \leq l$ and $\lfloor n\rfloor$ stands for the integer part of $n$, respectively, in one- and two-electron multi-center molecular integrals. These integrals needs to be calculated in spectroscopic accuracy in order to meaningful discussions on basis-set expansion methods, Born-Oppenheimer energy, vibrational frequency calculations. The difficulty of finding analytically closed form relations, however, for molecular integrals have more than two-center referred to as The bottleneck of quantum chemistry [10], have been greatest obstacle since Slater-type orbitals have no simple addition theorem; relations for products of two Slatertype orbitals centered on different positions not available in compact form [11].

The Slater-type orbitals are obtained by simplification of Laguerre functions in hydrogen-like orbitals [12] by keeping only the term of the highest power of $r$, for integer values of principal quantum number $n$ (ISTOs),

*albagci@univ-bpclermont.fr where $n \in \mathbb{N}^{+}, \Gamma(2 n+1)=(2 n)$ ! and it has been proved that they provide extra flexibility for closer variational description of atoms and molecules by considering the values of $n$ in more general set of number, namely positive real numbers (NSTOs), where $n \in \mathbb{R}^{+}$. The studies on the evaluation of molecular integrals, thus, are performed in two main group: those restrict the principal quantum number with integer values, which are practically used in nonrelativistic molecular electronic structure calculations $[13,14]$ and those free them from any specification but also reduce the area of applications only to investigation of atoms [15-20].

The multi-center molecular integrals over ISTOs can be evaluated by expansion of Slater-type orbitals through complete orthonormal basis functions to a new origin [2125] (see also references therein),

$$
\begin{aligned}
& \chi_{n l m}\left(\zeta, \vec{r}_{A}\right) \\
& =\lim _{N_{e} \rightarrow \infty} \sum_{n^{\prime} l^{\prime} m^{\prime}}^{N_{e}} V_{n l m, n^{\prime} l^{\prime} m^{\prime}}^{N_{e}}\left(\zeta, \vec{R}_{A B}\right) \chi_{n^{\prime} l^{\prime} m^{\prime}}\left(\zeta, \vec{r}_{B}\right) .
\end{aligned}
$$

or by expressing them as a finite linear combination of $B$ functions through Fourier transform [26-31]. However, infinite series representation formulas arising in expansion method require increasing upper limit of summation as much as possible to converge to exact values with sufficient decimals (the choice adopted as threshold for the total energy in nonrelativistic variational energy calculation is of order E-03 atomic units, therefore, constitute matrix elements should be accurate to E-10 atomic units) and presence of spherical Bessel functions brings computational difficulties in Fourier transform method since they provoke an oscillation [32-35].

The problem of multi-center integrals evaluation by the use of NSTOs even much more through insurmountable. The Slater type orbitals with noninteger principal quantum numbers do not have infinite series representation formulas; they can not be expanded via complete 


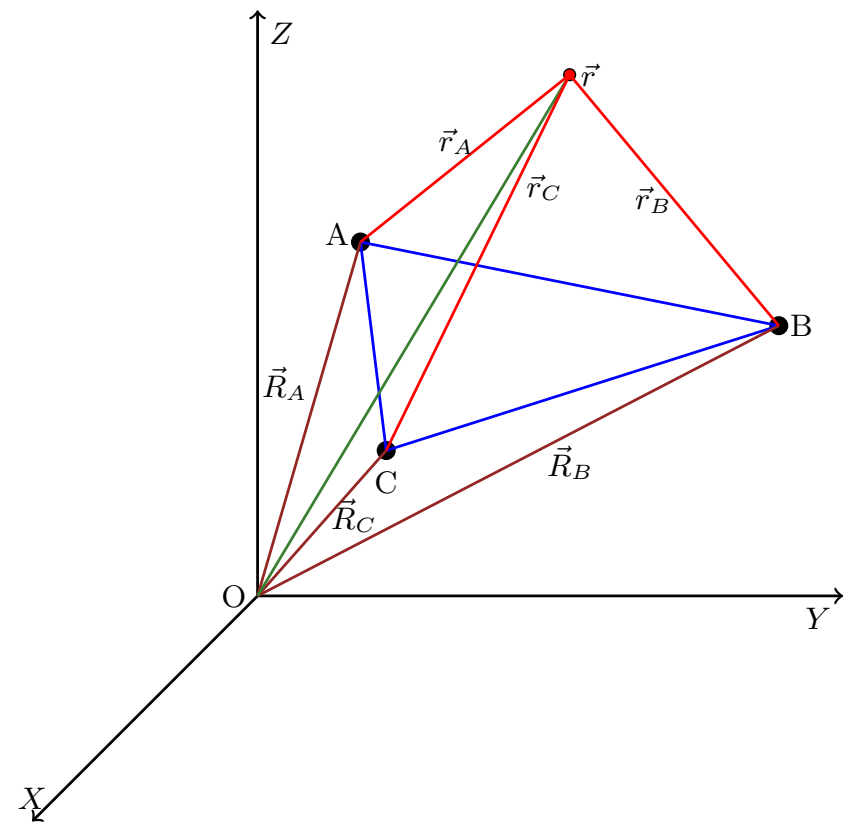

FIG. 1: Depiction of the coordinates for motion of an electron in the field of three stationary Coulomb centers, namely $A, B, C$, where $A=\left\{Z_{A}, Y_{A}, X_{A}\right\}$, $B=\left\{Z_{B}, Y_{B}, X_{B}\right\}, C=\left\{Z_{C}, Y_{C}, X_{C}\right\},\{Z, Y, X\}$ are the axes of Cartesian coordinates.

orthonormal basis functions since power series for a function such as $z^{\rho}, z \in \mathbb{C}$ and $\rho \in \mathbb{R} / \mathbb{N}_{0}$ are not analytic at the origin $[36,37]$, where the symbols $\mathbb{C}, \mathbb{R}$, $\mathbb{N}_{0}$ used to denote the sets of complex, real and natural numbers, respectively. It should be noted that, this also eliminates possibility of applying binomial expansion theorem in order to evaluate the two-center integrals, those are analytically closed form relations may obtain. Therefore, in mathematical point of view evaluation of multi-center molecular integrals using noninteger principal quantum numbers in Slater-type orbitals is an open question. It is far more than better representation of electronic wave-function in nonrelativistic electronic structure calculations it is also directly related with solution of the Dirac equation in algebraic approximation. The basis functions to be used in solution of matrix form of the Dirac equation are obtained analogously to L-spinors $[38,39]$ which are related to the Dirac hydrogenic solutions. Their explicit form include power functions $r^{\gamma}$,

$$
\gamma=\sqrt{\kappa^{2}-\frac{Z^{2}}{c^{2}}}
$$

with, $Z$ is nuclear charge, $c$ is speed of light, $\kappa=$ $\pm 1, \pm 2, \pm 2, \ldots$, respectively. They can only be represent by finite summation of Slater-type orbitals with noninteger principal quantum numbers.

In particular, the three-center integrals are the first set of multi-center integrals do not have analytically closed form relations. They have a fundamental importance in the study of molecular systems through $a b$-initio and density functional theory. They are central to the understanding of multi-center integrals. They have been commonly studied with methods presented above. They can also be evaluated through Neumann [40-43] and Laplace expansion [44-46] of Coulomb interaction in prolate spheroidal coordinates.

In a new approach the two-center integrals have been calculated by the authors for arbitrary values of parameters and quantum numbers via numerical integration techniques $[47,48]$. The new relativistic molecular auxiliary functions in prolate spheroidal coordinates are presented [48]. They are used to obtain compact form relations for two-electron integrals. Afterwards this idea adapted to calculate overlap integrals via Fourier transform formulas [49]. The same accuracy, 36-digits, is achieved in both methods. These are so far only known precise calculations for molecular integrals over NSTOs. Hence, they are used in this paper to produce benchmark values for three-center one-electron molecular Dirac integrals as a first time in the literature. The Mathematica programming language [50] is utilized for both analytical and numerical calculations.

\section{THREE-CENTER NUCLEAR ATTRACTION INTEGRALS}

Taking into account Fig. 1, where depiction of coordinates are given for one electron in a triangular conformation, the three-center nuclear attraction integrals are defined as follows,

$$
\begin{aligned}
& I_{n l m, n^{\prime} l^{\prime} m^{\prime}}\left(\zeta, \zeta^{\prime}, \vec{R}_{A B}, \vec{R}_{A C}\right) \\
& =\int \chi_{n l m}^{*}(\zeta, \vec{r}) \frac{1}{\left|\vec{r}-\vec{R}_{A C}\right|} \chi_{n^{\prime} l^{\prime} m^{\prime}}\left(\zeta^{\prime}, \vec{r}-\vec{R}_{A B}\right) d V,
\end{aligned}
$$

with, $A, B, C$ are three arbitrary points of the euclidian space, $\vec{R}_{A B}=\overrightarrow{A B}, \vec{R}_{A C}=\overrightarrow{A C}$.

The Neumann expansion for $1 /\left|\vec{r}-\vec{R}_{A C}\right|$ in prolate spheroidal coordinates $(\xi, \nu, \phi)$, where $1 \leq \xi \leq \infty,-1 \leq$ $\nu \leq 1,0 \leq \phi \leq 2 \pi[51]$,

$$
\begin{aligned}
\frac{1}{\left|\vec{r}-\vec{R}_{A C}\right|}=\frac{8 \pi}{R_{A B}} \sum_{L M} & (-1)^{M} \frac{(L-|M|) !}{(L+|M|) !} \\
\times \mathcal{P}_{L}^{|M|}\left(\xi_{<}\right) \mathcal{Q}_{L}^{|M|}\left(\xi_{>}\right) & \\
& \times Y_{L}^{M}\left(\nu_{C}, \phi_{C}\right) Y_{L}^{M}(\nu, \phi)^{*}
\end{aligned}
$$

here, $\mathcal{P}_{L}^{|M|}(\xi), \mathcal{Q}_{L}^{|M|}(\xi)$ are first and second kind associated Legendre functions [9], $\left\{\xi_{<}, \xi_{>}\right\}$refers to lesser and greater of $\left\{\xi_{c}, \xi\right\}$, respectively, is utilized in order to obtain expressions for the three-center nuclear attraction integrals over Slater-type orbitals, where the principal 
quantum numbers are free from specifications;

$$
\begin{aligned}
& I_{n l m, n^{\prime} l^{\prime} m^{\prime}}\left(\zeta, \zeta^{\prime}, \vec{R}_{A B}, \vec{R}_{A C}\right) \\
& =\frac{4 \sqrt{2 \pi}}{R_{A B}} \mathcal{N}_{n n^{\prime}}\left(\zeta, \zeta^{\prime}, R_{A B}\right) \sum_{L M}(-1)^{M} \frac{(L-|M|) !}{(L+|M|) !} A_{m m^{\prime}}^{M} \\
& \times Y_{L}^{M}\left(\nu_{C}, \phi_{C}\right)\left\{\mathcal{Q}_{L}^{|M|}\left(\xi_{C}\right) \mathcal{J}_{n l m, n^{\prime} l^{\prime} m^{\prime}}^{L M}\left(\zeta, \zeta^{\prime}, R_{A B}, \xi_{C}\right)\right. \\
& \left.\quad+\mathcal{P}_{L}^{|M|}\left(\xi_{C}\right) \mathcal{K}_{n l m, n^{\prime} l^{\prime} m^{\prime}}^{L M}\left(\zeta, \zeta^{\prime}, R_{A B}, \xi_{C}\right)\right\}
\end{aligned}
$$

here,

$$
\begin{aligned}
& \mathcal{N}_{n n^{\prime}}\left(\zeta, \zeta^{\prime}, R\right)= \\
& \frac{(2 \zeta)^{n+1 / 2}\left(2 \zeta^{\prime}\right)^{n^{\prime}+1 / 2}}{\left[\Gamma(2 n+1) \Gamma\left(2 n^{\prime}+1\right)\right]^{1 / 2}}\left(\frac{R}{2}\right)^{n+n^{\prime}+1}
\end{aligned}
$$

are the normalization constants and $A^{M}$ coefficients [52]

$$
\begin{array}{r}
A_{m m^{\prime}}^{M}=\frac{1}{\sqrt{2}}\left(2-\left|\eta_{m m^{\prime}}^{m-m^{\prime}}\right|\right)^{1 / 2} \delta_{M, \epsilon\left|m-m^{\prime}\right|} \\
+\frac{1}{\sqrt{2}} \eta_{m m^{\prime}}^{m+m^{\prime}} \delta_{M, \epsilon\left|m+m^{\prime}\right|},
\end{array}
$$

are the integration over azimuthal angle. The symbol $\epsilon$ may have the value \pm 1 and is determined by the product of the signs $m$ and $m^{\prime}$ (the sign of zero is regarded as positive). The symbols $\eta_{m m^{\prime}}^{m \pm m^{\prime}}$ may have the values \pm 1 and 0: if among the indices $m, m^{\prime}$ and $m \pm m^{\prime}$ there occurs a value equal to zero, then $\eta_{m m^{\prime}}^{m \pm m^{\prime}}$ is also zero; if all the indices differ from zero, $\eta_{m m^{\prime}}^{m \pm m^{\prime}}= \pm 1$ and the sign is determined by product of the signs $m, m^{\prime}, m \pm m^{\prime}$. Thus, the coefficients $A^{M}$ differ from zero only with the values $|M|=\left|m-m^{\prime}\right|,|M|=\left|m+m^{\prime}\right|$.

Since it is assumed axes of prolate spheroidal coordinate system centered on A, B substitutions in Eqs. $(4,5)$ can be written as follows [53],

$$
\begin{gathered}
\xi=\frac{r_{A}+r_{B}}{R_{A B}} ; \quad \nu=\frac{r_{A}-r_{B}}{R_{A B}}, \\
r_{A}=\frac{R_{A B}}{2}(\xi+\nu) ; \quad r_{B}=\frac{R_{A B}}{2}(\xi-\nu), \\
\xi_{C}=\frac{R_{A C}+R_{B C}}{R_{A B}} ; \quad \nu_{C}=\frac{R_{A C}-R_{B C}}{R_{A B}} .
\end{gathered}
$$

The $\mathcal{J}^{L M}, \mathcal{K}^{L M}$ integrals are the auxiliary functions and they are defined as,

$$
\begin{aligned}
& \mathcal{J}_{n l m, n^{\prime} l^{\prime} m^{\prime}}^{L M}\left(\zeta, \zeta^{\prime}, R_{A B}, \xi_{C}\right)=\int_{1}^{\xi_{C}} \int_{-1}^{+1}(\xi+\nu)^{n}(\xi-\nu)^{n^{\prime}} \\
& \times e^{-\xi\left[\frac{1}{2}\left(\zeta+\zeta^{\prime}\right) R_{A B}\right]-\nu\left[\frac{1}{2}\left(\zeta-\zeta^{\prime}\right) R_{A B}\right]} \\
& \times \overline{\mathcal{P}}_{l m}\left(\frac{1+\xi \nu}{\xi+\nu}\right) \overline{\mathcal{P}}_{l^{\prime} m^{\prime}}\left(\frac{1-\xi \nu}{\xi-\nu}\right) \\
& \times P_{L}^{|M|}(\xi) \overline{\mathcal{P}}_{L M}(\nu) d \xi d \nu
\end{aligned}
$$

$$
\begin{aligned}
\mathcal{K}_{n l m, n^{\prime} l^{\prime} m^{\prime}}^{L M} & \left(\zeta, \zeta^{\prime}, R_{A B}, \xi_{C}\right)=\int_{\xi_{C}}^{\infty} \int_{-1}^{+1}(\xi+\nu)^{n}(\xi-\nu)^{n^{\prime}} \\
\times & e^{-\xi\left[\frac{1}{2}\left(\zeta+\zeta^{\prime}\right) R_{A B}\right]-\nu\left[\frac{1}{2}\left(\zeta-\zeta^{\prime}\right) R_{A B}\right]} \\
\times & \overline{\mathcal{P}}_{l m}\left(\frac{1+\xi \nu}{\xi+\nu}\right) \overline{\mathcal{P}}_{l^{\prime} m^{\prime}}\left(\frac{1-\xi \nu}{\xi-\nu}\right) \\
& \times Q_{L}^{|M|}(\xi) \overline{\mathcal{P}}_{L M}(\nu) d \xi d \nu,
\end{aligned}
$$

where, $\overline{\mathcal{P}}_{l|m|}(x)$ are the normalized associated Legendre functions.

The auxiliary functions $\mathcal{J}^{L M}, \mathcal{K}^{L M}$ can be defined in a simpler form through relation given for product of two normalized associated Legendre functions centered on points $A, B$ in prolate spheroidal coordinates [40],

$$
\begin{gathered}
{\left[\left(\xi^{2}-1\right)\left(1-\nu^{2}\right)\right]^{\Lambda-\frac{\lambda+\lambda^{\prime}}{2}} \overline{\mathcal{P}}_{l \lambda}\left(\cos \theta_{A}\right) \overline{\mathcal{P}}_{l^{\prime} \lambda^{\prime}}\left(\cos \theta_{B}\right)} \\
=\sum_{\alpha=-(2 \Lambda-\lambda)}^{l} \sum_{\beta=\lambda^{\prime}}^{l^{\prime}} \sum_{q=0}^{\alpha+\beta-2 \Lambda-\lambda-\lambda^{\prime}} g_{\alpha \beta}^{q}\left(l \lambda, l^{\prime} \lambda^{\prime} ; \Lambda\right) \\
\times\left[\frac{(\xi \nu)^{q}}{(\xi+\nu)^{\alpha}(\xi-\nu)^{\beta}}\right] .
\end{gathered}
$$

which is obtained in explicit form by taking advantage of binomial expansion theorem,

$$
\begin{aligned}
(x+a)^{N_{1}}(x-a)^{N_{2}} & \\
& =\sum_{s=0}^{N_{1}+N_{2}} F_{s}\left(N_{1}, N_{2}\right) x^{N_{1}+N_{2}-s} a^{s},
\end{aligned}
$$

with,

$$
\cos \theta_{A}=\frac{1+\xi \nu}{\xi+\nu} ; \quad \cos \theta_{B}=\frac{1-\xi \nu}{\xi-\nu} .
$$

The coefficients $g_{\alpha \beta}^{q}$ occurring in Eq. (14) are determined by,

$$
\begin{gathered}
g_{\alpha \beta}^{q}\left(l \lambda, l^{\prime} \lambda\right)=g_{\alpha \beta}^{0}\left(l \lambda, l^{\prime} \lambda\right) F_{q}(\alpha+\lambda, \beta-\lambda) \\
g_{\alpha \beta}^{0}\left(l \lambda, l^{\prime} \lambda\right)=\sum_{s=0}^{\lambda}(-1)^{s} F_{s}(\lambda) D_{\alpha+2 \lambda-2 s}^{l \lambda} D_{\beta}^{l^{\prime} \lambda},
\end{gathered}
$$

$$
\begin{aligned}
D_{\beta}^{l \lambda}=\frac{1}{2^{l}}(-1)^{(l-\beta) / 2} & {\left[\frac{2 l+1}{2} \frac{F_{l}(l+\lambda)}{F_{\lambda}(l)}\right]^{1 / 2} } \\
& \times F_{(l-\beta) / 2}(l) F_{\beta-\lambda}(l+\beta),
\end{aligned}
$$

where, $\lambda=|m|, \lambda^{\prime}=\left|m^{\prime}\right|$ and the quantities $F_{s}\left(N, N^{\prime}\right)$ are the generalized binomial coefficients. They are given as,

$$
F_{s}\left(N, N^{\prime}\right)=\sum_{s^{\prime}}(-1)^{s^{\prime}} F_{s-s^{\prime}}(N) F_{s^{\prime}}\left(N^{\prime}\right)
$$


with, $\frac{1}{2}[(s-N)+|s-N|] \leq s^{\prime} \leq \min (s, N)$ and $F_{s}(N)$ are binomial coefficients indexed by $N$ and $s$ is usually written $\left(\begin{array}{c}N \\ s\end{array}\right)$, respectively.

The Eqs. $(12,13)$ are, therefore, obtained as follows,

$$
\begin{gathered}
{\left[\begin{array}{c}
\mathcal{J}_{n l m, n^{\prime} l^{\prime} m^{\prime}}^{L M}\left(\zeta, \zeta^{\prime}, R_{A B}, \xi_{C}\right) \\
\mathcal{K}_{n l m, n^{\prime} l^{\prime} m^{\prime}}^{L M}\left(\zeta, \zeta^{\prime}, R_{A B}, \xi_{C}\right)
\end{array}\right]=\sum_{\alpha \beta q} g_{\alpha \beta}^{q}\left(l \lambda, l^{\prime} \lambda^{\prime} ; \Lambda\right)} \\
\times\left[\begin{array}{c}
\mathcal{J}_{n-\alpha, q}^{L M, n^{\prime}-\beta}\left(\zeta, \zeta^{\prime}, R_{A B}, \xi_{C}\right) \\
\mathcal{K}_{n-\alpha, n^{\prime}-\beta}^{L M, q}\left(\zeta, \zeta^{\prime}, R_{A B}, \xi_{C}\right)
\end{array}\right],
\end{gathered}
$$

with,

$$
\begin{aligned}
& {\left[\begin{array}{c}
\mathcal{J}_{n-\alpha, n^{\prime}-\beta}^{L \Lambda, q}\left(\zeta, \zeta^{\prime}, R_{A B}, \xi_{C}\right) \\
\mathcal{K}_{n-\alpha, n^{\prime}-\beta}^{L \Lambda, q}\left(\zeta, \zeta^{\prime}, R_{A B}, \xi_{C}\right)
\end{array}\right]} \\
& =\int_{\left[\begin{array}{c}
\xi_{C} \\
\infty
\end{array}\right]}^{1} \int_{-1}^{1}(\xi \nu)^{q}(\xi+\nu)^{n-\alpha}(\xi-\nu)^{n^{\prime}-\beta} \\
& \times e^{-\xi\left[\frac{1}{2}\left(\zeta+\zeta^{\prime}\right) R_{A B}\right]-\nu\left[\frac{1}{2}\left(\zeta-\zeta^{\prime}\right) R_{A B}\right]} \\
& \times\left[\begin{array}{c}
\mathcal{P}_{L}^{|\Lambda|}(\xi) \\
\left.\mathcal{Q}_{L}^{|\Lambda|}(\xi)\right]
\end{array}\right] \overline{\mathcal{P}}_{L|\Lambda|}(\nu) d \xi d \nu
\end{aligned}
$$

There are no known convergent series representation formulas, free from specifications on parameters for power functions such as $(\xi+\nu)^{N_{1}},(\xi-\nu)^{N_{2}},\left\{N_{1}, N_{2}\right\} \in \mathbb{R}$, yet and that poses an obstacle to analytically reduce the $\mathcal{J}^{L \Lambda, q}, \mathcal{K}^{L \Lambda, q}$ auxiliary functions to one variable $w_{\mu}^{q}, L_{\mu}^{q}, k_{\mu}^{q}$ auxiliary functions introduced in [42]. Thus, the solution should be obtained on the basis of numerical methods. Note that, taking advantage of binomial expansion method for terms containing the angular part of Slater-type orbitals in order to simplify the expressions increases the number of integrals should be numerically calculated. In Mathematica programming language instead of using Eq. (20), direct computation of Eqs. (12, 13) are faster. The given relations for auxiliary functions in Eq. (21) are calculated by using different expressions of Legendre polynomials and compared according to computational time in Fig. (2) as sample. The discussions on results are made in the next section.

\section{RESULTS AND DISCUSSIONS}

The literature currently, lack of benchmark values for multi-center integrals when Slater-type orbitals are used. Recently, a robust numerical Global-adaptive strategy with Gauss-Kronrod extension has been applied for two-center integrals through prolate spheroidal coordinates and fourier transform method in [47-49]. Benchmark results have been presented for them. The hermitian properties are thus, represented correctly free from specification on quantum numbers, orbital parameters and internuclear distances. In this study it is extended for solution of three-center integrals. The algo- rithm described in [47] has been incorporated into a computer program written in the Mathematica programming language with the included numerical computation packages for solving Eqs. $(6,12,13)$. The Mathematica programming language can handle approximate real numbers with any number of digits and it is suitable for benchmark evaluation. It is also provides a uniquely integrated and automated environment for parallel computing. It is allows us to compute the formulas including summations using all cores of PC effectively via ParallelSum command instead of Sum. Note that, in this study all results are given in atomic units (a.u.). The calcula-

TABLE I: The values for auxiliary functions defined in Eq.(21) with $p_{1}=\frac{1}{2}\left(\zeta+\zeta^{\prime}\right) R_{A B}, p_{2}=\frac{1}{2}\left(\zeta-\zeta^{\prime}\right) R_{A B}$ and $N_{1}, N_{2} \in \mathbb{R}^{+}$.

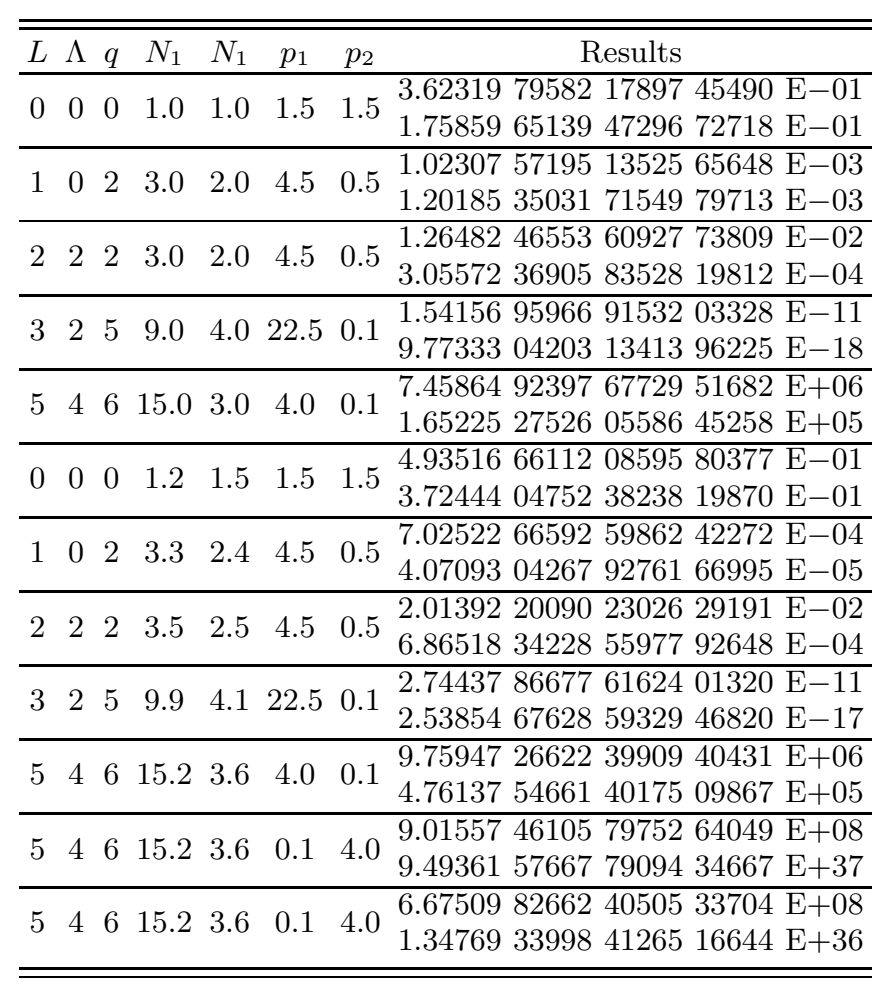

tion results are presented in Tables I-VII and Fig.2 for arbitrary values of quantum numbers, orbital parameters and internuclear distances. The comparisons are made with expansion methods which are given for expansion of wave function in Eq. (2) and for charge density expansion to same center by following formula [54-56]

$$
\begin{aligned}
& \rho_{n l m, n^{\prime} l^{\prime} m^{\prime}}\left(\zeta, \vec{r} ; \zeta^{\prime}, \vec{r}\right) \\
& =\sum_{l^{\prime \prime}=\left|l-l^{\prime}\right|}^{l+l^{\prime}} \sum_{m^{\prime \prime}=-l^{\prime \prime}}^{l^{\prime \prime}} W_{n l m, n^{\prime} l^{\prime} m^{\prime}, n+n^{\prime}-1 l^{\prime \prime} m^{\prime \prime}}\left(\zeta, \zeta^{\prime}, z\right) \\
& \times \chi_{n+n^{\prime}-1 l^{\prime \prime} m^{\prime \prime}}(z, \vec{r}) .
\end{aligned}
$$


They are useful to reduce the three-center integrals to basic nuclear attraction integrals,

$$
J_{\kappa \lambda \tau}\left(z, \vec{R}_{B C}\right)=\frac{1}{\sqrt{4 \pi}} \int \chi_{\kappa \lambda \tau}^{*}\left(z, \vec{r}_{B}\right) \frac{1}{r_{C}} d v_{1} .
$$

The Eqs. $(2,22)$ are used to transform the wave function centered at $A$ to a wave function centered at $B$ then, to transform the charge density centered on same positions to a single wave function, respectively. Here, $z=\zeta+$ $\zeta^{\prime}, \vec{R}_{B C}=\overrightarrow{A C}$. The resulting basic nuclear attraction integrals are calculated by the following formula, [57],

$$
\begin{gathered}
J_{\kappa \lambda \tau}(z, \vec{R})= \\
\frac{2^{\kappa}}{2 \lambda+1} \sqrt{\frac{2}{z} \frac{\Gamma(\kappa+\lambda+2)}{\sqrt{\Gamma(2 \kappa+1)}}} \frac{1}{(z R)^{\lambda+1}} \\
\times\left(1-\frac{\Gamma(\kappa+\lambda+2, z R)}{\Gamma(\kappa+\lambda+2)}+\frac{(z R)^{2 \lambda+1} \Gamma(\kappa-\lambda+1, z R)}{\Gamma(\kappa+\lambda+2)}\right) \\
\times Y_{\lambda, \tau}(\theta, \phi),
\end{gathered}
$$

where, $\Gamma(n, m)$ are incomplete gamma functions [9].

In Fig. 2 the Eq. (21) is investigated according to computational time in Mathematica programming language. Mathematica includes all the common special functions of mathematical physics. It also provides easy way of computing them precisely. Here, explicit formula (EF) [58],

$$
\begin{gathered}
\overline{\mathcal{P}}_{l \lambda}(x)=\left(1-x^{2}\right)^{\frac{\lambda}{2}} \sum_{k} b_{l \lambda}^{k} x^{l-\lambda-2 k}, \\
b_{l \lambda}^{k}=\frac{1}{2^{l}}\left[\frac{2 l+1}{2 F_{\lambda}(l) F_{\lambda}(l+\lambda)}\right]^{\frac{1}{2}} \\
\times(-1)^{k} F_{k}(\lambda+k) F_{l-k}(2 l-2 k) F_{l-\lambda-2 k}(l-k),
\end{gathered}
$$

where, $0 \leq k \leq E\left[\frac{l-\lambda}{2}\right]$, recurrence relation formula (RF) [59] of Legendre polynomials are compared with Mathematica built-function (MF) LegendreP $[\mathbf{n}, \mathbf{m}, \mathbf{x}]$. They are presented with red, blue, green lines in Fig. 2, respectively. It can be seen from this figure, the direct use of Mathematica buit-function, given for computing of Legendre polynomials, in numerical integration of Eq. (21) provide the results faster then explicit or recurrence relation formulas.

The results for calculation of Eq. (21) are also presented in Table I with integer and noninteger values of principal quantum numbers. The first, second rows are obtained from calculation $\mathcal{J}_{N_{1} N_{2}}^{L \Lambda, q}$ and $\mathcal{K}_{N_{1} N_{2}}^{L \Lambda, q}$ functions, respectively. The auxiliary functions $w_{\mu}^{q}, L_{\mu}^{q}, k_{\mu}^{q}$ defined in [42] for three-center integrals are special case of Eq. (21). Hence, we believe an importance of present the results for general form of Eq. (21).

The results for Eqs. (12, 13) and Eq. (20) are presented in Tables II, III. They are given in first, second rows for $\mathcal{J}_{n l m, n^{\prime} l^{\prime} m^{\prime}}^{L M}$ and $\mathcal{K}_{n l m, n^{\prime} l^{\prime} m^{\prime}}^{L M}$ auxiliary functions, respectively. Note that, the Eq. (20) is only differ from

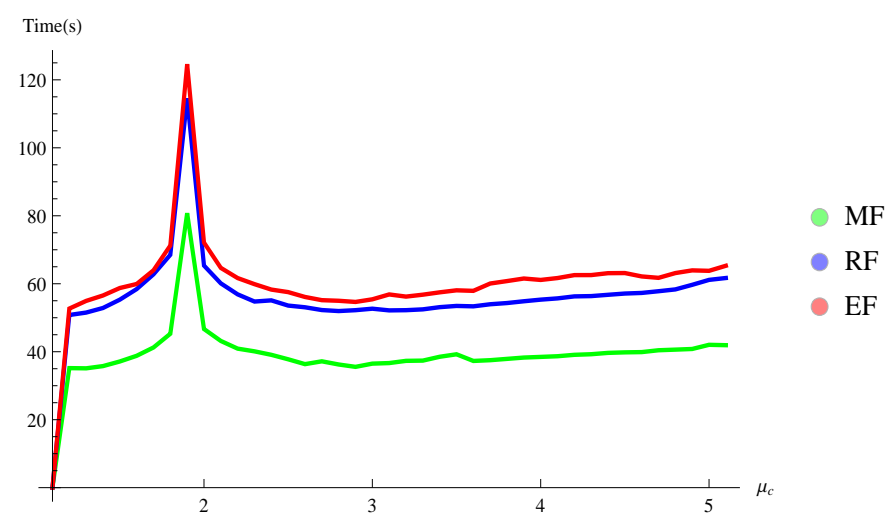

FIG. 2: CPU time for computation of $\mathcal{J}_{N_{1} N_{2}}^{L \Lambda, q}$ auxiliary function in Eq.(21) according to methods used for calculation of the Legendre polynomials, where,

Mathematica built-function (MF), recurrence relation formula (RF), explicit formula ( $\mathrm{EF})$ and, $L=3, \Lambda=1$, $q=0, N_{1}=3, N_{2}=2, p_{1}=2.5, p_{2}=1.5$.

Eqs. (12, 13) in that, the normalized associated Legendre polynomials on right-hand side are expanded via Eq. (14) and the numerical global adaptive method is performed to remaining parts. Performing the calculations in Mathematica programming language for such formulas contain summations is disadvantageous in terms of calculation time. The results in Tables II, III shows that, numerical Global adaptive method with Gauss-Kronrod extension can be used for computation of Eqs. $(12,13)$ which is eliminate necessity applying binomial expansion theorem.

In Tables IV, V, VI the results obtained for threecenter integrals are presented for upper limit of summation $L$ is $L=30$. The correct digits are underlined. The digits in bold indicate the convergence property of used method. In first and second rows benchmark results obtained from numerical global adaptive method and results those found in the literature are presented, respectively. Later rows are the results obtained from expansion of the wave-function method and they are in complete agreement with ones from E. Şahin (personal communication), where the upper imit of summations $N_{e}$ are given in parenthesis. The expansion of the wave-function method is tested up to upper limit of summation $N_{e}$, $N_{e}=160$. It is observed that, the results hardly convergent with 10-digits for given quantum numbers and orbital parameters in table V. In other tables the convergence remains between 5-digits and 10-digits. Note that, it is necessary to take into account eight summation and four of them should be infinite in expansion of the wave-function method if NSTOs are used. The values presented in Tables IV, V, VI for STOs clearly demonstrate pointlessness of such an attempt.

On the other hand in our previous papers $[47,48]$ it have been proved that the numerical Global adaptive method with Gauss-Kronrod extension is able to give benchmark 
values. In particular for Table VII the results are presented for different upper limit of summation $L$ appears in Eq. (6). They differs from upper limit of summation $N_{e}$ used in expansion of STOs in that they are given in brackets. Convergence property of Eq. (6) is examined in this table. It is found that, by increasing the upper limit of summation the results are convergence to exact values. The results obtained up to upper limit of summation $L$ is $L=40$. It is achieved to 25 -digits accuracy by determining the upper limit of summation $L$ is $L=30$ accordingly, there is no necessity of performing calculations with upper limit of summation higher than $L=30$ unless more precise results required for a given values of parameters. It should be point out that, the summation appears in Eq. (6) should not be regarded as having same characteristic with summation arising in expansion of STOs. It is based on expansion of spherical harmonics which have form a complete set of orthonormal functions. Any square-integrable function can be expanded as a linear combination of spherical harmonics. The convergence problems arising in expansion NSTOs can not exist in our method. Without any computational difficulty by increasing the upper limit of summation $L$ can be achieved to desired accuracy rapidly.

\section{ACKNOWLEDGEMENT}

A.B. acknowledges funding for a postdoctoral research fellowship from innov@pole: the Auvergne Region and FEDER.
[1] C. C. J. Roothaan, Rev. Mod. Phys. 23, 69 (1951).

[2] T. Kato, Commun. Pure. Appl. Math. 10, 151 (1957).

[3] S. Agmon, Lectures on Exponential Decay of Solution of Second-Order Elliptic Equations: Bounds on Eigenfunctions of N-Body Schrödinger Operations (Princeton University Press, Princeton, NJ, 1982).

[4] J. C. Slater, Phys. Rev. 36, 57 (1930).

[5] R. G. Parr and H. W. Joy, J. Chem. Phys. 26, 424 (1957).

[6] E. U. Condon and G. H. Shortley, The Theory of Atomic Spectra (Cambridge University Press, Cambridge, 1935).

[7] E. O. Steinborn and K. Ruedenberg, Adv. Quantum Chem. 7, 1 (1973).

[8] M. A. Blanco, M. Florez, and M. Bermejo, J. Mol. Struct. (THEOCHEM) 419, 19 (1997).

[9] M. Abramowitz and I. A. Stegun, Handbook of Mathematical Functions with Formulas, Graphs, and Mathematical Tables (Dover Publications, New York, 1972).

[10] R. S. Mulliken and C. C. J. Roothaan, Proc. Natl. Acad. Sci. 45, 394 (1959).

[11] A. Bouferguene and H. W. Jones, J. Chem. Phys. 109, 5718 (1998).

[12] D. J. Willock, Molecular Symmetry, Appendix 9: The Atomic Orbitals of Hydrogen (John Wiley\&Sons Ltd, Chichester, UK, 2009).

[13] A. Bouferguene, M. Fares and P. E. Hoggan, Int. J. Quantum Chem. 57, 801 (1996).

[14] J. Fernández Rico, R. López, A. Aguado, I. Ema and G. Ramírez, Int. J. Quantum Chem. 81, 148 (2001).

[15] T. Koga, K. Kanayama and A. J. Thakkar, Int. J. Quantum Chem. 62, 1 (1997).

[16] T. Koga and K. Kanayama, J. Phys. B: At. Mol. Opt. Phys. 30, 1623 (1997).

[17] T. Koga and K. Kanayama, Chem. Phys. Lett. 266123 (1997).

[18] T. Koga, J. M. García de la Vega and B. Miguel, Chem. Phys. Lett. 283, 97 (1998).

[19] T. Koga, T. Shimazaki and T. Satoh, J. Mol. Struct. (Theochem), 496, 95 (2000) 95.

[20] M. Erturk, Comput. Phys. Commun, 194, 59 (2015).

[21] M. P. Barnett and C. A. Coulson, Phil. Trans. R. Soc. London A 243, 221 (1951).
[22] F. E. Harris and H. H. Michels, J. Chem. Phys. 43, 165 (1965).

[23] I. I. Guseinov, J. Chem. Phys. 69, 4990 (1978).

[24] I. I. Guseinov, Int. J. Quantum Chem. 81, 126 (2001).

[25] A. Bouferguene, J. Phys. A: Math. Gen. 38, 2899 (2005).

[26] E. Filter and E. O. Steinborn, Phys. Rev. A 18, 1 (1978).

[27] E. Filter and E. O. Steinborn, J. Math. Phys. 19, 79 (1978).

[28] E. J. Weniger and E. O. Steinborn, J. Chem. Phys. 78, $6121(1983)$.

[29] J. Grotendorst and E. O. Steinborn, J. Comput. Phys. 61, 195 (1985).

[30] E. O. Steinborn, H. H. H. Homeier and E. J. Weniger, J. Mol. Struct. (THEOCHEM) 260, 207 (1992).

[31] H. H. H. Homeier, E. J. Weniger and E. O. Steinborn, Comput. Phys. Commun. 72, 269 (1992).

[32] H. Safouhi and P. E. Hoggan, J. Comput. Phys. 155, 331 (1999).

[33] H. Safouhi, J. Comput. Phys. 165, 473 (2000).

[34] L. Berlu and H. Safouhi, J. Phys. A: Math. Gen. 36, 11267 (2003).

[35] L. Berlu and H. Safouhi, J. Phys. A: Math. Gen. 36, 11791 (2003).

[36] E. J. Weniger, J. Phys. A: Math. Theor. 41, 425207 (2008).

[37] E. J. Weniger, J. Math. Chem. 50, 17 (2012).

[38] I. P. Grant, H. M. Quiney, Phys. Rev. A 62, 022508 (2000).

[39] I. P. Grant, Relativistic Quantum Theory of Atoms and Molecules (Springer, New York, 2007).

[40] I. I. Guseinov, J. Chem. Phys. 65, 4718 (1976).

[41] J. Fernández Rico, R. López, and G. Ramírez, J. Chem. Phys. 97, 7613 (1992).

[42] F. E. Harris, Int. J. Quant. Chem. 88, 701 (2002).

[43] K. Peuker and M. B. Ruiz, J. Math. Chem. 43, 701 (2008).

[44] P. J. Roberts, J. Chem. Phys. 50, 1381 (1969).

[45] J. Fernández Rico, R. López, and G. Ramírez, J. Chem. Phys. 91, 4204 (1989).

[46] J. Fernández Rico, R. López, and G. Ramírez, J. Chem. Phys. 94, 5032 (1991). 
[47] A. Bağcı and P. E. Hoggan, Phys. Rev. E 89, 053307 (2014).

[48] A. Bağcı and P. E. Hoggan, Phys. Rev. E 91, 023303 (2015).

[49] H. J. Silverstone, J. Phys. Chem. 118, 11971 (2014).

[50] http://www.wolfram.com/mathematica/.

[51] K. Ruedenberg, J. Chem. Phys. 19, 1459 (1951).

[52] I. I. Guseinov, J. Phys. B 3, 1399 (1970).

[53] G. Gordadse, Zeitschrift für Physik 96, 542 (1935).

[54] I. I. Guseinov and B. A. Mamedov, Int. J. Quantum Chem. 78, 146 (2000).

[55] I. I. Guseinov, Int. J. Quantum Chem. 90, 980 (2002).

[56] I. I. Guseinov, J. Math. Chem. 42, 415 (2007).

[57] I. I. Guseinov and B. A. Mamedov, Theor. Chem. Acc. 108, 21 (2002).

[58] I. I. Guseinov, J. Mol. Struct. (THEOCHEM) 335, 17 (1995).

[59] NIST Digital Library of Mathematical Functions, http://dlmf.nist.gov/, Release 1.0.9 of 2014-08-29. 
TABLE II: The values for auxiliary functions defined in Eqs. $(12,13)$ and Eq. (20) with $p_{1}=\frac{1}{2}\left(\zeta+\zeta^{\prime}\right) R_{A B}$, $p_{2}=\frac{1}{2}\left(\zeta-\zeta^{\prime}\right) R_{A B}$ and $n, n^{\prime} \in \mathbb{N}^{+}$.

\begin{tabular}{|c|c|c|c|c|c|c|c|c|c|c|}
\hline$L$ & $M$ & $n$ & $l$ & $m$ & $n^{\prime}$ & $l^{\prime}$ & $m^{\prime}$ & $p_{1}$ & $p_{2}$ & Eqs. $(12,13)$ and Eq. (20) \\
\hline 0 & 0 & 1.0 & 0 & 0 & 1.0 & 0 & 0 & 1.5 & 2.5 & 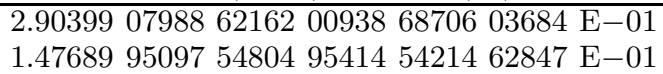 \\
\hline 2 & 0 & 3.0 & 0 & 0 & 2.0 & 0 & 0 & 0.01 & 2.5 & 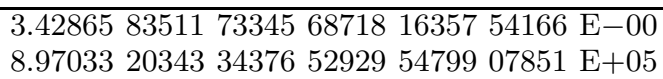 \\
\hline 5 & 0 & 9.0 & 0 & 0 & 4.0 & 0 & 0 & 22.5 & 0.1 & 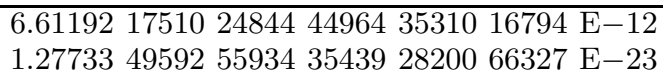 \\
\hline 10 & 0 & 12.0 & 0 & 0 & 8.0 & 0 & 0 & 40.0 & 0.001 & $\begin{array}{lllllll}1.73471 & 85107 & 61691 & 61997 & 46481 & 63897 & \mathrm{E}-20 \\
1.87470 & 00384 & 84104 & 39018 & 32142 & 95122 & \mathrm{E}-41\end{array}$ \\
\hline 6 & 0 & 6.0 & 4 & 0 & 5.0 & 3 & 0 & 0.001 & 0.001 & $\begin{array}{lllllll}2.25821 & 42942 & 16571 & 75095 & 60318 & 57985 & \mathrm{E}+46 \\
2.48928 & 81670 & 28192 & 48883 & 41015 & 71291 & \mathrm{E}+56\end{array}$ \\
\hline 1 & 0 & 2.0 & 1 & 1 & 2.0 & 1 & 1 & 0.01 & 0.01 & 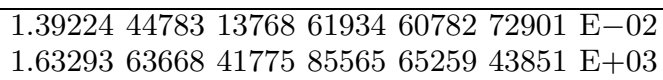 \\
\hline 3 & 2 & 10.0 & 4 & 4 & 8.0 & 2 & 2 & 0.1 & 9.0 & 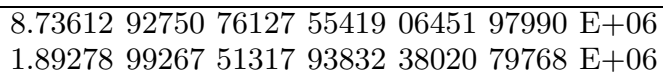 \\
\hline
\end{tabular}


TABLE III: The values for auxiliary functions defined in Eqs. $(12,13)$ and Eq. $(20)$ with $p_{1}=\frac{1}{2}\left(\zeta+\zeta^{\prime}\right) R_{A B}$, $p_{2}=\frac{1}{2}\left(\zeta-\zeta^{\prime}\right) R_{A B}$ and $n, n^{\prime} \in \mathbb{R}^{+}$.

\begin{tabular}{|c|c|c|c|c|c|c|c|c|c|c|}
\hline$L$ & $M$ & $n$ & $l$ & $m$ & $n^{\prime}$ & $l^{\prime}$ & $m^{\prime}$ & $p_{1}$ & $p_{2}$ & Eq. $(16,17)$ and Eq.(19) \\
\hline 0 & 0 & 1.1 & 0 & 0 & 1.5 & 0 & 0 & 1.5 & 1.5 & $\begin{array}{lllllll}4.09565 & 63231 & 31448 & 87039 & 55147 & 40491 & \mathrm{E}-01 \\
2.93614 & 23789 & 04133 & 04860 & 14608 & 03840 & \mathrm{E}-01\end{array}$ \\
\hline 1 & 0 & 1.3 & 0 & 0 & 1.8 & 0 & 0 & 0.5 & 0.1 & $\begin{array}{lllllll}3.33243 & 32418 & 64279 & 67130 & 02542 & 67866 & \mathrm{E}-01 \\
1.02388 & 22819 & 29861 & 91096 & 69560 & 06751 & \mathrm{E}-02\end{array}$ \\
\hline 2 & 0 & 3.2 & 0 & 0 & 2.4 & 0 & 0 & 0.01 & 2.5 & 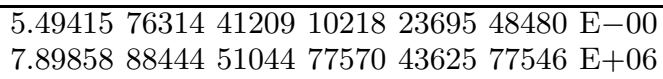 \\
\hline 5 & 0 & 9.5 & 0 & 0 & 4.8 & 0 & 0 & 22.5 & 0.1 & 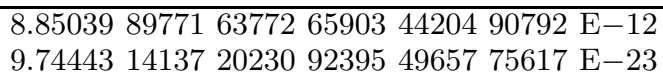 \\
\hline 1 & 0 & 3.3 & 1 & 0 & 2.6 & 1 & 0 & 2.3 & 4.5 & $\begin{array}{lllllll}1.87161 & 11340 & 32480 & 14502 & 70364 & 65188 & \mathrm{E}-00 \\
6.65100 & 22118 & 49834 & 63694 & 23667 & 20358 & \mathrm{E}-01\end{array}$ \\
\hline 6 & 0 & 5.9 & 4 & 0 & 6.1 & 3 & 0 & 0.001 & 0.001 & 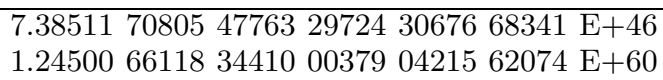 \\
\hline 3 & 0 & 8.8 & 4 & 3 & 10.3 & 3 & 3 & 0.01 & 0.01 & 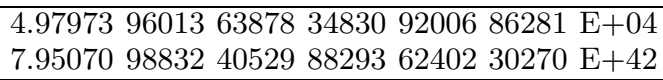 \\
\hline 3 & 2 & 4.5 & 3 & 3 & 3.5 & 2 & 1 & 1.5 & 1.5 & 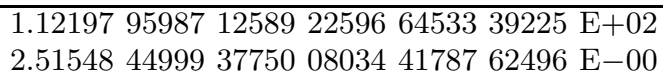 \\
\hline 3 & 2 & 10.5 & 4 & 4 & 8.1 & 2 & 2 & 0.1 & 9.0 & $\begin{array}{lllllll}1.10758 & 39108 & 61744 & 40668 & 98531 & 33050 & \mathrm{E}+07 \\
1.32690 & 39575 & 82905 & 48879 & 15023 & 14344 & \mathrm{E}+29\end{array}$ \\
\hline
\end{tabular}

TABLE IV: The values for three-center integrals, where position of the nuleus A, B, C in cartesian coordinates $\{X, Y, Z\}: \mathrm{A}=\{0,0,0\}, \mathrm{B}=\{0,0,-2.0143\}, \mathrm{C}=\{0,0,-4.1934\}$, respectively.

\begin{tabular}{|c|c|c|c|c|c|c|c|c|}
\hline$n$ & $l$ & $m$ & $\zeta$ & $n^{\prime}$ & $l^{\prime}$ & $m^{\prime}$ & $\zeta^{\prime}$ & Results \\
\hline 1.0 & 0 & 0 & 1.24 & 1.0 & 0 & 0 & 5.67 & 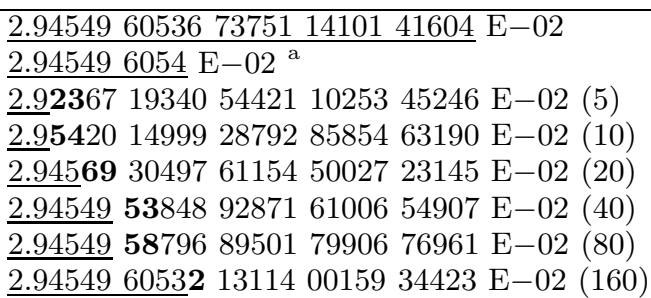 \\
\hline 1.2 & 0 & 0 & 1.24 & 1.2 & 0 & 0 & 5.67 & 3.7481124252417417187567668 E-02 \\
\hline 1.3 & 0 & 0 & 1.24 & 1.3 & 0 & 0 & 5.67 & $\overline{4.1629170512 \quad 3679353772 \quad 11297}$ E-02 \\
\hline 1.0 & 0 & 0 & 1.24 & 2.0 & 0 & 0 & 1.61 & 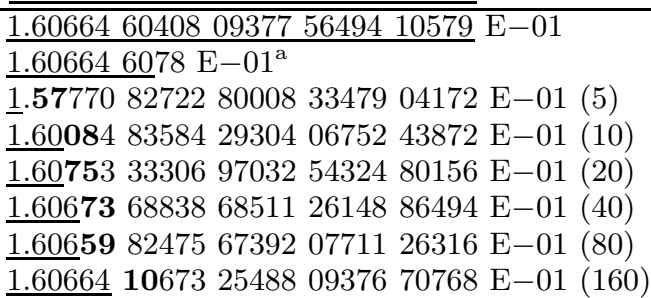 \\
\hline 1.1 & 0 & 0 & 1.24 & 2.1 & $\overline{0}$ & $\overline{0}$ & 1.61 & $\overline{1.6976382691195733604553004 \mathrm{E}-01}$ \\
\hline 1.2 & 0 & 0 & 1.24 & 2.2 & 0 & 0 & 1.61 & $\overline{1.78213088676001908900 \quad 15372} \mathrm{E}-01$ \\
\hline 1.3 & 0 & 0 & 1.24 & 2.3 & 0 & 0 & 1.61 & $\overline{1.8600031133 \quad 3394268781 \quad 62339}$ E-01 \\
\hline
\end{tabular}

a $[11]$ 
TABLE V: The values for three-center integrals, where $\zeta=\zeta^{\prime}=2.0$; position of the nuleus A, B, C in cartesian coordinates $\{X, Y, Z\}: \mathrm{A}=\{0,0,0\}, \mathrm{B}=\{0,0,6\}, \mathrm{C}=\{0,0,-7\}$, respectively.

\begin{tabular}{|c|c|c|c|c|c|c|}
\hline$n$ & $l$ & $m$ & $n^{\prime}$ & $l^{\prime}$ & $m^{\prime}$ & Results \\
\hline 2.0 & 0 & 0 & 2.0 & 0 & 0 & 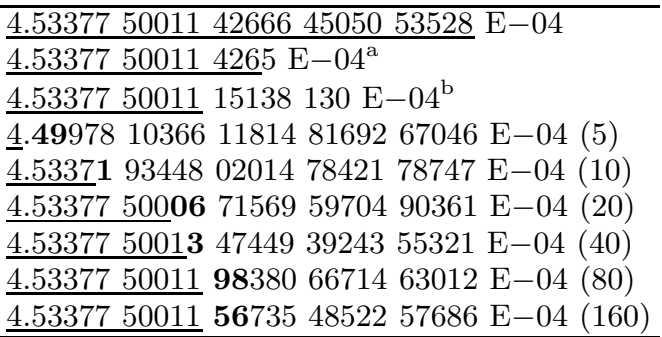 \\
\hline 2.1 & 0 & 0 & 2.1 & 0 & 0 & $\overline{5.5182466271} 984902031429459 \mathrm{E}-04$ \\
\hline 2.2 & 0 & 0 & $\overline{2.2}$ & 0 & 0 & $\underline{6.66626 \quad 60479988639311416105} \mathrm{E}-04$ \\
\hline 2.3 & 0 & 0 & 2.3 & 0 & 0 & $\overline{7.995475377228315 \quad 4333278068}$ E-04 \\
\hline 2.0 & 1 & 0 & 2.0 & 0 & 0 & $\begin{array}{ll}6.499892518910135 \quad 1058999466 \\
6.4998925189 \quad 1011 \mathrm{E}-04^{\mathrm{a}}\end{array}$ \\
\hline 2.1 & 1 & 0 & 2.1 & 0 & 0 & $\overline{7.90609} 20760 \quad 42277 \quad 16143 \quad 10272 \mathrm{E}-04$ \\
\hline 2.2 & 1 & 0 & 2.2 & 0 & 0 & 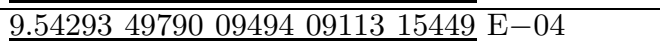 \\
\hline 2.3 & 1 & 0 & 2.3 & 0 & 0 & $\overline{1.14342806668602344617 \quad 17750} \mathrm{E}-03$ \\
\hline 2.0 & 1 & 0 & 2.0 & 1 & 0 & $\begin{array}{llll}9.69666 \quad 68121 & 64802 \quad 1068205157 & \mathrm{E}-04 \\
9.6966668121 & 6477 \mathrm{E}-04^{\mathrm{a}} & \\
\end{array}$ \\
\hline 2.1 & 1 & 0 & 2.1 & 1 & 0 & 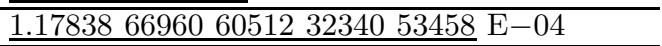 \\
\hline 2.2 & 1 & 0 & 2.2 & 1 & 0 & $1.42089425543492639305 \quad 88294$ E-04 \\
\hline 2.3 & 1 & $\overline{0}$ & 2.3 & 1 & 0 & $1.7005457496599945152055390 \mathrm{E}-03$ \\
\hline
\end{tabular}

a [41]

b $[43]$

TABLE VI: The values for three-center integrals, where $\zeta=\zeta^{\prime}=1.6$; position of the nuleus $\mathrm{A}, \mathrm{B}, \mathrm{C}$ in cartesian coordinates $\{X, Y, Z\}: \mathrm{A}=\{0,0,0\}, \mathrm{B}=\{0,0,3\}, \mathrm{C}=\{0,0,-3\}$, respectively.

\begin{tabular}{|c|c|c|c|c|c|c|}
\hline$n$ & $l$ & $m$ & $n^{\prime}$ & $l^{\prime}$ & $m^{\prime}$ & Results \\
\hline 2.0 & 0 & 0 & 2.0 & 0 & 0 & 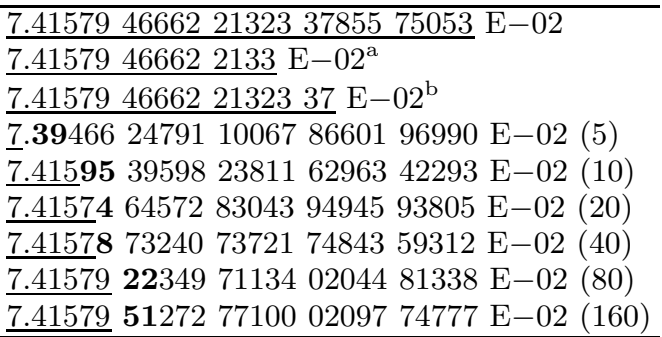 \\
\hline 2.1 & 0 & 0 & 2.1 & 0 & 0 & $\overline{7.92564} 04306464545128728675 \mathrm{E}-02$ \\
\hline 2.2 & 0 & 0 & $\overline{2.2}$ & 0 & 0 & $8.4244584406538039691857249 \mathrm{E}-02$ \\
\hline 2.3 & 0 & 0 & 2.3 & 0 & 0 & $\overline{8.90946 \quad 2936956705 \quad 1839486907}$ E-02 \\
\hline 2.0 & 1 & 0 & 2.0 & 0 & 0 & 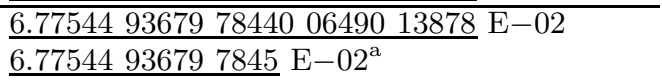 \\
\hline 2.1 & 1 & 0 & 2.1 & 0 & 0 & $\overline{7.1024782121126689950925056}$ E-02 \\
\hline 2.2 & 1 & 0 & 2.2 & 0 & 0 & $\overline{7.3966478499315629124965250} \mathrm{E}-02$ \\
\hline 2.3 & 1 & 0 & 2.3 & 0 & 0 & $\overline{7.65548} 51830 \quad 78592 \quad 48565 \quad 84851 \quad \mathrm{E}-02$ \\
\hline 2.0 & 1 & 0 & 2.0 & 1 & 0 & $\begin{array}{ll}6.75368 \quad 18945 \quad 49233 \quad 3633486913 \\
6.75368 \quad 18945 \quad 4926 \quad \mathrm{E}-02^{\mathrm{a}}\end{array}$ \\
\hline 2.1 & 1 & 0 & 2.1 & 1 & 0 & $6.91775 \quad 52123 \quad 726129633496130 \mathrm{E}-02$ \\
\hline 2.2 & 1 & 0 & 2.2 & 1 & 0 & 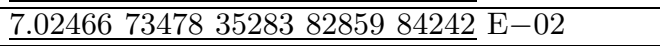 \\
\hline 2.3 & 1 & 0 & 2.3 & 1 & 0 & $\underline{7.07260} 26896462340813755301 \mathrm{E}-02$ \\
\hline
\end{tabular}

a [41]

b [43] 
TABLE VII: The values for three-center integrals, where $\zeta=3.6, \zeta^{\prime}=1.6$; position of the nuleus A, B, C in cartesian coordinates $\{X, Y, Z\}: \mathrm{A}=\{0,0,0\}, \mathrm{B}=\{0,0,3\}, \mathrm{C}=\{3,0,3\}$, respectively.

\begin{tabular}{|c|c|c|c|c|c|c|}
\hline$n$ & $l$ & $m$ & $n^{\prime}$ & $l^{\prime}$ & $m^{\prime}$ & Results \\
\hline 2.0 & 0 & 0 & 2.0 & 0 & 0 & 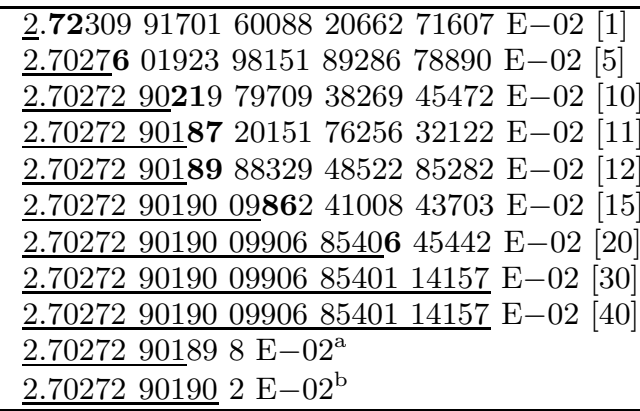 \\
\hline 2.1 & 0 & 0 & 2.1 & 0 & 0 & 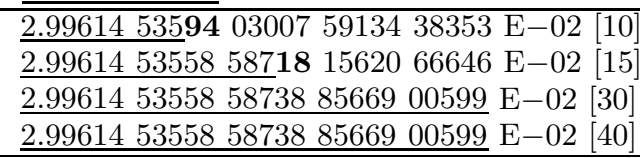 \\
\hline 2.2 & 0 & 0 & 2.2 & 0 & 0 & 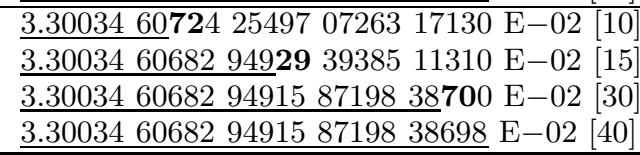 \\
\hline 2.3 & 0 & 0 & 2.3 & 0 & 0 & $\left.\begin{array}{llllll}3.61354 & 34150 & 29555 & 79331 & 66393 & \mathrm{E}-02\end{array}\right]$ \\
\hline 2.0 & 1 & 0 & 2.0 & 0 & 0 & 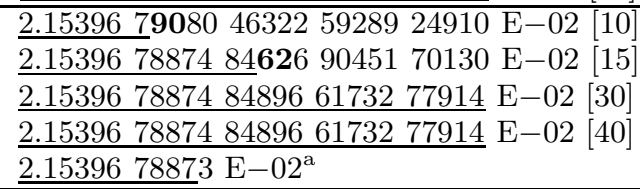 \\
\hline 2.1 & 1 & 0 & 2.1 & 0 & 0 & 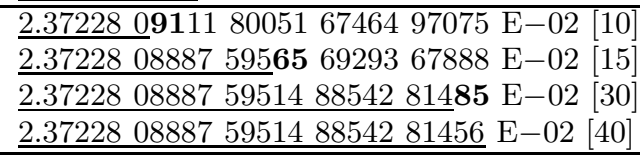 \\
\hline 2.2 & 1 & 0 & 2.2 & 0 & 0 & $\begin{array}{lllllll}2.59362 & 19462 & 42909 & 94539 & 77789 & \mathrm{E}-02 & {[10]} \\
2.59362 & 19225 & 73538 & 25384 & 87321 & \mathrm{E}-02 & {[15]} \\
2.59362 & 19225 & 73080 & 19834 & 68030 & \mathrm{E}-02 & {[30]} \\
2.59362 & 19225 & 73080 & 19834 & 67975 & \mathrm{E}-02 & {[40]}\end{array}$ \\
\hline 2.3 & 1 & 0 & 2.3 & 0 & 0 & $\begin{array}{lllllllll}2.81580 & 26527 & 44329 & 75185 & 31334 & \mathrm{E}-02 & {[10]} \\
2.81580 & 26286 & 47036 & 51507 & 08634 & \mathrm{E}-02 & {[15]} \\
2.81580 & 26286 & 46091 & 20634 & 90123 & \mathrm{E}-02 & {[30]} \\
2.81580 & 26286 & 46091 & 20634 & 90048 & \mathrm{E}-02 & {[40]}\end{array}$ \\
\hline 2.0 & 1 & 0 & 2.0 & 1 & 0 & 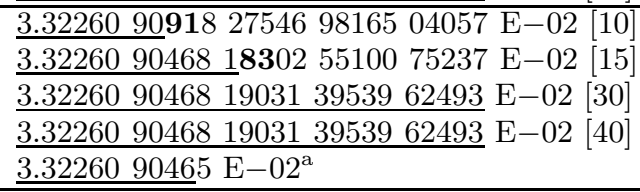 \\
\hline 2.1 & 1 & 0 & 2.1 & 1 & 0 & 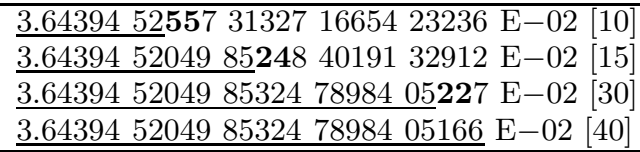 \\
\hline 2.2 & 1 & 0 & 2.2 & 1 & 0 & 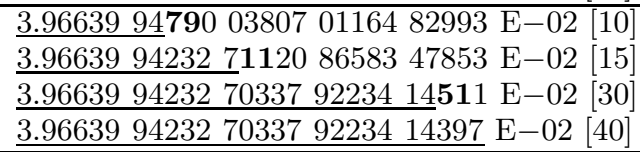 \\
\hline 2.3 & 1 & 0 & 2.3 & 1 & 0 & 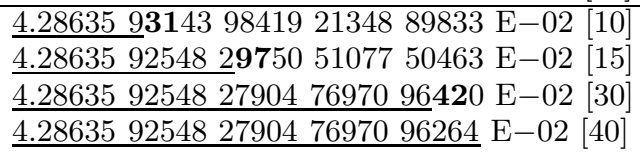 \\
\hline
\end{tabular}

